\title{
Evaluation of the performance of designed coaxial antennas for hyperthermia using simulation and experimental methods
}

\author{
Ayo Z IBITOYE ${ }^{a}$, Obande C OGESE ${ }^{a}$, Margaret B ADEDOKUN ${ }^{b}$, Muhammad Y HABEEBU ${ }^{a}$, Ephraim O NWOYE , \\ Adebayo M AWEDA ${ }^{a}$ \\ ${ }^{a}$ Department of Radiation Biology and Radiotherapy, College of Medicine, University of Lagos, Nigeria \\ ${ }^{b}$ Department of Physics, University of Lagos, Nigeria \\ ${ }^{c}$ Department of Biomedical Engineering, College of Medicine, University of Lagos, Nigeria \\ *E-mail address: azibitoye@cmul.edu.ng
}

\begin{abstract}
Introduction: Antenna geometries and tissue properties affect microwave energy distributions during microwave ablation procedures. There is paucity information on the potential of antenna fabricated from a thick semi-rigid coaxial cable in the field of microwave thermal therapy. This study aimed at comparing the performance of two dual-slot antennas designed from different semi-rigid coaxial cables for the ablation of a liver tumour using numerical simulation and experimental validation methods.

Materials and Methods: COMSOL Multiphysics software was used for designing dual-slot antennas and as well as to evaluate microwave energy deposition and heat distribution in the liver tissue. Experimental validations were conducted on the ex-vivo bovine livers to validate the simulation results.

Results: Thick antenna developed in this study produced a higher sphericity index, larger ablation diameter and reduced backward heating along the antenna shaft than the existing one. The experimental validation results also indicate significant differences between the two antennas in terms of ablation diameters $(p=0.04)$, ablation lengths $(p=0.02)$ and aspect ratios $(\mathrm{p}=0.02)$.

Conclusion: Based on the findings in this study, antenna fabricated from a thick coaxial cable has a higher potential of localizing microwave energy in the liver than conventional antennas.
\end{abstract}

Key words: liver tumour; coaxial dual-slot antenna; microwave ablation; hyperthermia.

\section{Introduction}

Microwave ablation (MWA) is a form of thermal ablation therapy using the application of heat to destroy tumors in different anatomical sites. ${ }^{1-2}$ Microwave ablation has been clinically tried over a range of tumours located in the lung, ${ }^{3}$ kidney, ${ }^{4}$ liver ${ }^{5-6}$ breast, ${ }^{7}$ pancreas, ${ }^{8}$ and prostate. ${ }^{9}$ During MWA, microwave energy propagation is isotropically radiative and its absorption in tissue is primarily due to dielectric losses. Propagation and deposition of electromagnetic energy in biological tissues are determined by their dielectric permittivity, effective conductivity and water content. Microwave energy is effectively absorbed in frequencies of $915 \mathrm{MHz}$ and $2450 \mathrm{MHz}$ by biological tissues leading to the oscillation of polar molecules due to dipole action. Heat is generated as a result of the oscillation of polar molecules in the biological tissues leading to a rise in temperature. The degree of rising in temperature depends on the applied input power and duration of microwave application. ${ }^{10}$

Microwave antennas play vital roles in the distribution of heat in biological tissues. A good antenna for microwave ablation therapy should be able to aid heat energy distribution locally into the targeted tissue volume. It must be able to ablate large tumour volume with reduced backward heating along the antenna shaft. Whereas the occurrence of backward heating along the antenna shaft is due to inherent unbalanced coaxial cable structure. To achieve those features, antennas such as a monopole, dipole, slot, choked, sleeved, helical antennas have been proposed. ${ }^{11-14}$ Some of these antennas still exhibit some limitations, especially backward heating effects and high reflection coefficient. ${ }^{15}$ Also, most of the reported antennas had been designed and fabricated from coaxial cables of diameter in the range between 1.5 and $2.5 \mathrm{~mm}$. The efficiency of the monopole, single-slot, dual-slot and sleeved antennas fabricated from a thin semi-rigid coaxial cable $(2.21 \mathrm{~mm}$ in diameter) has been studied compared and reported elsewhere ${ }^{16}$ with paucity information about antennas designed from thicker semi-rigid coaxial cables. Previous studies have shown that antennas designed from such a range of diameters are characterized by inadequate power handling capacity, which normally results in backward heating. ${ }^{17-18}$ Therefore, there is a 


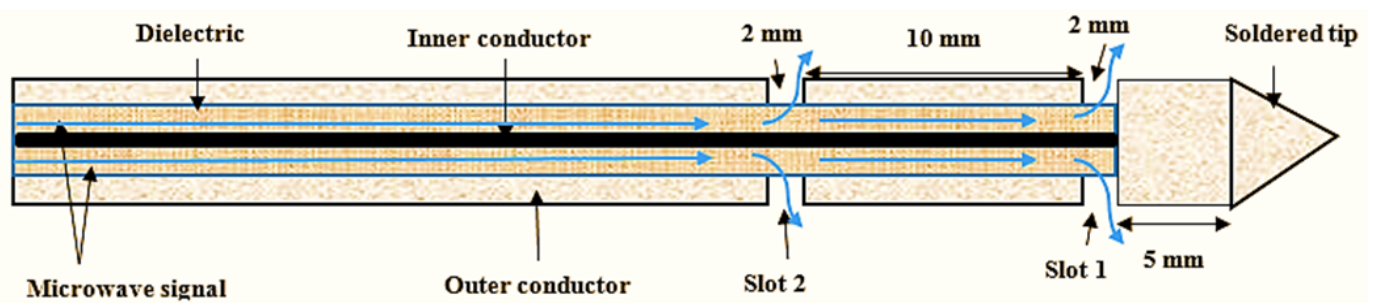

Figure 1. Schematic diagram of typical dual-slot antenna design. Two antennas A and B were simulated and fabricated from coaxial cables of different thicknesses but the same prototype and dimensions shown in the figure.

need to develop an antenna that can withstand high power input for a long duration without damaging it. The antenna must also exhibit a reduced backward heating effect to preserve sensitive organs along the antenna insertion path.

Computer modelling, which has played a crucial role in designing suitable antennas in the field of microwave technology, is being used to achieve the aforementioned objective. Computer simulation is a quick, convenient and inexpensive tool for evaluating, isolating, and optimizing of promising devices for prototyping. ${ }^{19-24}$ It is also an effective method in understanding the interactions between microwave energy and biological tissues. Finite element method (FEM) and finite-difference time-domain (FDTD) has been adopted in the antennas' geometry specifications and evaluation of heat transfer resulting from interactions of the microwave with biological tissues. Theoretically, microwave energy propagation and absorption energy in tissue is governed by Maxwell's equations while Pennes' bio-heat equation governs the temperature profile in tissue during ablation and these have been discussed extensively in the literature. ${ }^{19-20}$ To establish the study's validity, an experimental validation method that includes fabrication of the antenna from the selected coaxial cables, ex vivo experimentation and analysis of the obtained data must be compared with the simulation results. Thus, in this study, we employed computer simulation and experimental validation methods to evaluate the efficiency of two antennas fabricated from different semi-rigid coaxial cables meant for microwave ablation therapy.

\section{Materials and Methods}

\section{Design and Simulation of Antennas}

Finite element methods (FEMs) package in COMSOL Multiphysics v 4.4 (Stockholm, Sweden) software was used to design and simulate antennas $\mathrm{A}$ and $\mathrm{B}$ from semi-rigid coaxial cables of $025^{\prime \prime}(6.35 \mathrm{~mm})$ and $0.085^{\prime \prime}(2.21 \mathrm{~mm})$ in diameters respectively. The software has the facilities for geometry specifications, studying heat transfer as a result of electromagnetic field distribution in the biological tissues. Parameters such as ablation size, ablation shape, power deposited into the tissue, temperature profile, necrosis parameter, power reflection coefficient, specific absorption rate (SAR) were predicted with the aid of this software. The inner conductor of the coaxial cables was made from silver-covered copper and dielectric material made from a solid polytetrafluoroethylene (PTFE) while the outer shield was made from a bare copper tube. The geometries and prototype of the simulated antennas are shown in Figure 1. These semirigid coaxial cables were chosen for this study to match the $\mathrm{N}$ type connector of a microwave generator available in our laboratory. Dual slot antenna was chosen in this study because it has been reported as having the ability to localize microwave energy in the liver without additional metallic sleeve or choke compared to other antennas. 14,25

Radiofrequency and heat modules in the software were used to study microwave energy distributions in the liver tissue and the bio-heat effects. ${ }^{26}$ The axially symmetric model was used to minimize the computation time while maintaining excellent resolution and the full 3-D nature of the fields. The electric and magnetic fields associated with the time-varying transverse electromagnetic (TEM) wave generated by the microwave source propagating in a coaxial cable in the $\mathrm{z}$-direction is expressed in 2-D axially symmetric cylindrical coordinates as in Equations 1-4.

$\boldsymbol{E}(r)=e_{r} \frac{\boldsymbol{c}}{r} e^{j(\omega t-k z)}$ Eq. 1

$\boldsymbol{H}(r)=e_{\varphi} \frac{C}{\boldsymbol{Z r}} e^{j(\omega t-k z)}$

$P_{a v}=\int_{r_{i}}^{r_{o}} \operatorname{Re}\left(\frac{1}{2} \boldsymbol{E} \times \boldsymbol{H}\right) 2 \pi r d r=e_{z} \pi \frac{c^{2}}{Z} \operatorname{In}\left(\frac{r_{o}}{r_{i}}\right)$

with $C=\sqrt{\frac{Z P_{\text {in }}}{\pi \cdot \ln \left(r_{0} / r_{i}\right)}}$

where $\boldsymbol{E}$ is the electric field ( $\mathrm{V} \mathrm{m}-1), \boldsymbol{H}$ is the magnetic field strength $\left(\mathrm{A} \mathrm{m}{ }^{-1}\right), r_{o}$ and $r_{i}$ are the dielectric's inner and outer radii, respectively $(\mathrm{m}), \mathrm{P}_{\text {in }}$ is the input power $(\mathrm{W}), \mathrm{Z}$ is the wave impedance in the dielectric of the coaxial cable $(\Omega), \mathrm{z}$ is the direction of propagation, and $\mathrm{r}, \phi$, and $\mathrm{z}$ are cylindrical coordinates centred on the axis of the coaxial cable. $P_{a v}$ is the time-averaged power flow in the cable, and $\omega$ denotes the angular frequency. The propagation constant, $k=2 \pi / \lambda$, and $\lambda$ is the wavelength in the medium. In tissue, the electric field has a finite axial component (r) whereas the magnetic field is purely in the azimuthal direction ( $\mathrm{z}$ ). Thus, the antennas were modelled using an axisymmetric transverse magnetic (TM) formulation.

The most significant effect of an electromagnetic field applied is the conversion of microwave energy to thermal energy. The temperature profile in tissue during ablation is obtained by solving a Pennes' bioheat Equation 5: 
$\rho c \frac{d T}{d t}=\nabla \cdot k \nabla T+Q-Q_{p}+Q_{m}$

Eq. 5

where $\rho\left(\mathrm{kg} / \mathrm{m}^{3}\right)$ is the tissue density, $\mathrm{c}(\mathrm{J} / \mathrm{kg} \cdot \mathrm{k})$ is the specific heat of the tissue, $\mathrm{k}(\mathrm{W} / \mathrm{mK})$ is thermal conductivity, $\mathrm{T}(\mathrm{K})$ is the temperature, $\mathrm{Q}\left(\mathrm{W} / \mathrm{m}^{3}\right)$ is the absorbed electromagnetic energy, $Q_{p}\left(\mathrm{~W} / \mathrm{m}^{3}\right)$ is the heat loss due to microvascular blood perfusion, and $Q_{m}\left(\mathrm{~W} / \mathrm{m}^{3}\right)$ is the metabolic heat generation. Generally, $Q_{m}$ is ignored, as its magnitude is substantially smaller than other terms in this equation. The absorbed electromagnetic energy, $\mathrm{Q}$, is computed from the electromagnetic field distribution in tissue and is given as:

$Q=1 / 2 \sigma|E|^{2}$

Eq. 6

When normalized by tissue density, $\rho, \mathrm{Q}$ is referred to as specific absorption rate (SAR) therefore Equation 5 can be rewritten as:

$\rho c \frac{d T}{d t}=\nabla \cdot k \nabla T-Q_{p}+S A R+Q_{m}$

Eq. 7

Thermal damage due to heating is a function of the degree of temperature elevation, tissue properties, ablation duration and configuration of the applicator (antenna) used to deliver the microwave energy. A fraction of necrotic tissue (thermal injury), $\phi$, is expressed as:

$\phi=1-e^{(-\Omega(\mathrm{t}))}$

Eq. 8

where $\Omega(t)$ is the survival fraction of cells in tissue exposed to elevated temperature as:

$\Omega(t)=\int_{0}^{t} A \exp \left\{\frac{E_{a}}{R T(t)}\right\} d t$

Where $\mathrm{A}(1 / \mathrm{s})$ is the frequency factor, $\mathrm{E}_{\mathrm{a}}(\mathrm{J} / \mathrm{mol})$ is the activation energy required to transform tissue from normal to damaged state, $\mathrm{R}(\mathrm{J} / \mathrm{mol} \cdot \mathrm{k})$ is the universal gas constant and $\mathrm{T}$ (k) is the absolute temperature of the tissue.

The tissue was modelled geometrically as a cylinder of 50 $\mathrm{mm}$ by $95 \mathrm{~mm}$ height and assumes to be homogeneous. The liver's properties were taken from the literature and compared with the software built-in values. ${ }^{27,28}$ The horizontal $\mathrm{z}$-axis was oriented along the longitudinal axis of the antenna and the vertical $r$-axis was oriented along the radial direction.

In the electromagnetic wave propagation analysis, the scattering boundary condition was set on the surface, which means that the boundary does not disturb the electromagnetic field distribution. The microwave signal was set at the upper end of the antenna (a dielectric component of the coaxial cable). The microwave energy propagating in each antenna was characterized by transverse electromagnetic fields while transverse magnetic fields in tissue. No phase change occurs, no energy exchange through the outer surface and no chemical reactions occur within the tissue.

The model uses a frequency-domain problem formulation with the complex-valued azimuthal component of the magnetic field as the unknown. The asymmetric finite-element model was discretized using the adaptive triangular element with a maximum element size of $3 \mathrm{~mm}$ and the Lagrange quadratic was used to approximate microwave power absorbed. The external surface of the tissues acts as a boundary for the computational domain. A low reflecting boundary was thermally isolated. Metallic components were assumed to be perfect electric conductors (PEC) and the coaxial dielectric was assumed to be lossless PTFE. Perfectly matched layers were assumed for all exterior boundaries. Input power was set to 50 $\mathrm{W}$ at $2.45 \mathrm{GHz}$ in all the simulations. Electric field, temperature, necrosis, SAR distributions were computed and analysed for all the tissues. The power reflection coefficient and power dissipation into the tissue were also quantified. The lesion size and shape were calculated using the $52.0^{\circ} \mathrm{C}$ isothermal contours after $10 \mathrm{~min}$ with an input power of $50 \mathrm{~W}$, since, at this temperature coagulation and cell death are possible.

\section{Experimental validation}

Microwave energy was delivered by using a $2.45 \mathrm{GHz}$ solidstate microwave generator (SAIREM SAS, Neyron-Cedex, France), which has adjustable features for the input powers and time. Ex vivo experimentation was performed on the bovine liver tissue obtained from a local abattoir. Semi-rigid coaxial cables RG401/U and RG405/U (Pasternack Enterprise Inc, Irvine) of $0.25^{\prime \prime}(6.35 \mathrm{~mm})$ and $0.085^{\prime \prime}(2.21 \mathrm{~mm})$ in diameters for the antennas A and B respectively were used. Antennas A and B were fabricated from $50 \Omega$ semi-rigid coaxial cables to conform to the geometrical specifications described in the simulated method section (Figure 1). Fifteen bovine liver samples were ablated using the input power of $50 \mathrm{~W}$ for 3,5 and 10 minutes using these antennas. Based on our previous experience, semi-rigid coaxial cables could withstand input power of about $50 \mathrm{~W}$ for a long duration for effective ablation without damaging the applicator. Five ablation processes were conducted for the input power and ablation duration. After the ablation procedure, each tissue was sliced along the axis of the antenna to evaluate maximum dimensions of the coagulation region in axial and radial cross-section after 3 hours. Ablation diameter, ablation length and aspect ratio were physically measured with a highly accurate graduated ruler. Sliced ablated tissues were also captured by a high-resolution camera for further analysis. For all the ablated tissue; ablation lengths, ablation diameters and sphericity indices were computed. The sphericity index was calculated from the quotient of $r_{1}{ }^{2}$ and $r_{2}{ }^{2}$. Where $r_{1}$ and $r_{2}$ are the radii of short and long- axis of the ablated tissue respectively. ${ }^{29-30}$

ImageJ software from National Institute of Health $(\mathrm{NIH}$, USA) was used for the analysis of images generated from simulation and experimental validation. A student's t-test was used to compare the statistically significant difference between the antennas. A value of $\mathrm{p}<0.05$ was considered to be statistically significant. 


\section{Results and Discussion}

\section{Simulation Results}

The simulation results using dual-slot antennas designed from different semi-rigid coaxial cables are presented in Table $\mathbf{1}$ and Figures 2-4.

Table 1 shows the obtained values from simulation procedures using thick antenna $\mathrm{A}$ and thin antenna $\mathrm{B}$ from different coaxial cables. From the table, the reflection coefficient was decreased by $16.4 \%$ when using antenna A compared with the existing antenna B. This is an indication that antenna A exhibits better impedance matching with the liver tissue than antenna B tissue. Power deposition was also increased by $7.3 \%$ as a result of the low reflection coefficient. Maximum temperature which could have resulted in excessive heating of the coaxial cable was reduced by $15.5 \%$. Based on these results, antenna A has the higher potential of heating tissue using high input powers without generating excessive heat that will have negative effects on the performance of the antenna. Antenna A has its SAR reduced by $45.0 \%$. This significant percentage difference may be assumed to be connected to the excess SAR along the shaft of antenna B which results in the generation of heat along its track. Ablation length along the antenna shaft in antenna A was reduced by $15.9 \%$ when compared with antenna B. This is an indication that antenna A can reduce backward heating along the antenna shaft during the MWA procedures. Where tissue preservation is necessary along the antenna shaft is expedient, antenna A will also be more suitable than antenna B. Ablation diameter (short-axis) of antenna A increased by $13.0 \%$ in comparison with antenna B. As a result of this, tumours of larger diameters or localized tumours bigger than $4 \mathrm{~cm}$ can be ablated with antenna A better than antenna B. The sphericity index of antenna A was $15.9 \%$ more than that of antenna B. This signifies an increase in the localization of microwave energy at the tip of antenna A than antenna B.

Figure 2 shows a variation of temperature distribution versus insertion depth using the input power of $50 \mathrm{~W}$ for 10 minutes. The peak temperature difference between the antennas at the depth of $67 \mathrm{~mm}$ from the proximal end of the antennas was about $44^{\circ} \mathrm{C}$ with the antenna B produced $235^{\circ} \mathrm{C}$. A reduced temperature at the surface of antenna A shows its potential application to preserve normal tissue at its point of insertion during ablation procedures. In Figure 3, temperature distributions at different isothermal levels are presented. Backward heating along the shaft of the antenna $\mathrm{A}$ is more reduced than antenna $\mathrm{B}$ at different temperature levels.
Table 1. Simulation results produced by antennas A and B

\begin{tabular}{lccc}
\hline \hline Parameter & Antenna A & Antenna B & \% difference \\
\hline Reflection Coefficient (dB) & -7.1 & -6.1 & -16.4 \\
Power deposited density (W) & 39.5 & 36.8 & 7.3 \\
Max Temp (@10 min) ${ }^{\circ} \mathrm{C}$ & 191.8 & 227.1 & -15.5 \\
Max SAR (kW/kg) & 6.1 & 11.1 & -45.0 \\
Short-axis (mm) & $39.0^{\mathrm{a}}$ & $38.6^{\mathrm{c}}$ & $13.0^{\mathrm{e}}$ \\
Long-axis(mm) & $43.6^{\mathrm{b}}$ & $46.4^{\mathrm{d}}$ & $-15.9^{\mathrm{f}}$ \\
Sphericity index & 0.80 & 0.69 & 15.9 \\
\hline \hline
\end{tabular}

$a=$ ablation length of antenna $A, b=$ ablation diameter of antenna $A$,

$c=$ ablation diameter of antenna $B, d=$ ablation length of antenna $B$,

$e=\%$ difference in the ablation diameters, $f=\%$ difference in the ablation lengths

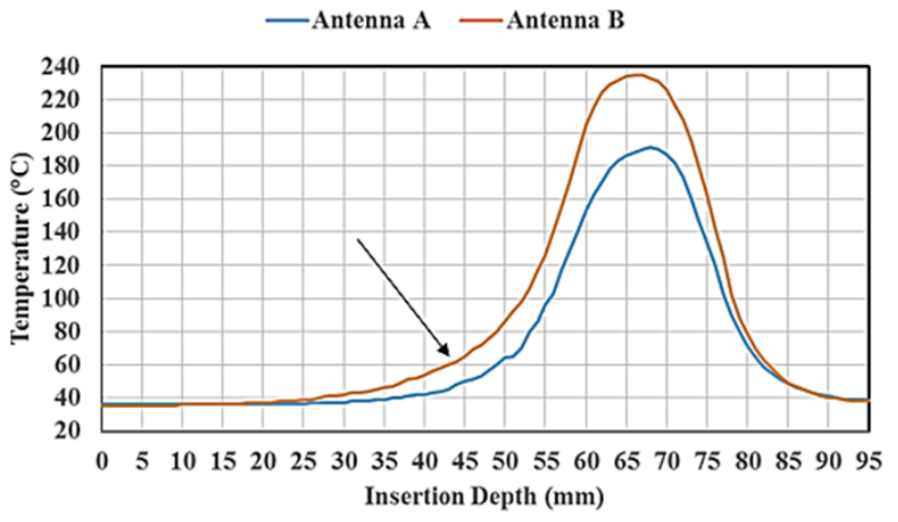

Figure 2. Variations in temperature versus insertion depth from the proximal end of the antennas. The arrow in the figure indicates that antenna A produced less backward heating than antenna $B$ due to reduced temperature generated during the microwave ablation procedure. Antenna B produced higher temperatures at the depths beyond $25 \mathrm{~mm}$ than the antenna $\mathrm{A}$.

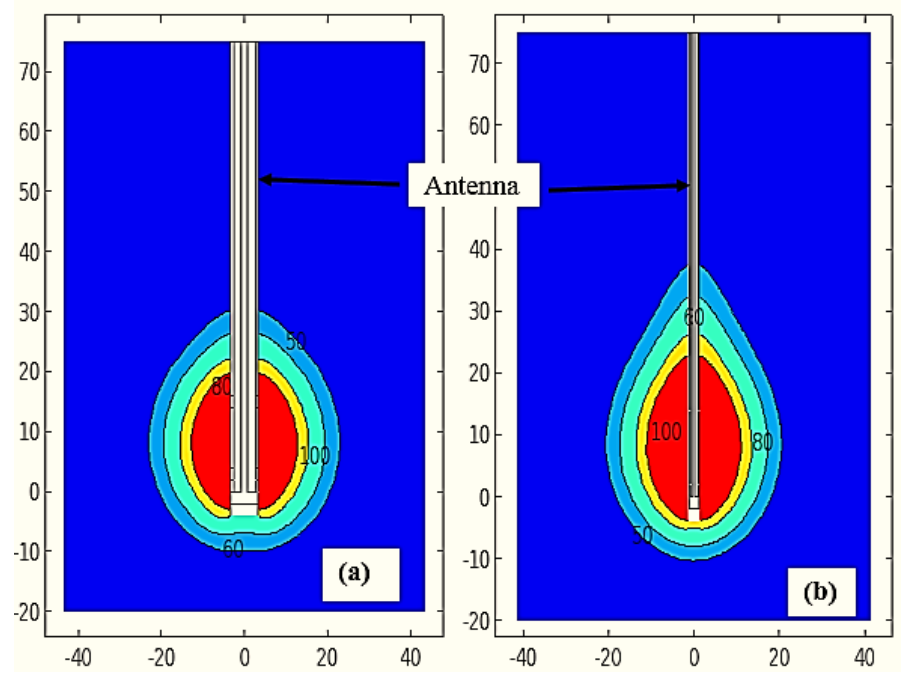

Figure 3. Isothermal distributions in ablated tissue in (a) produced by antenna $A$ and (b) produced by antenna $B$. The shape of the isothermal distribution produced at different temperatures in (a) is more spherical than those in (b). Backward heating along the antenna shaft was lowered in (a) than (b). 
Figure 4 shows 3-D necrotic regions and the corresponding isothermal temperature contours $\left(52.0^{\circ} \mathrm{C}\right)$ at which the probability of cellular destruction is $63 \% .^{8}$ As shown in the figure, antenna A produced more localized microwave energy than antenna B, therefore, making it (Antenna A) capable of destroying tumours that display spherical morphology.

\section{Experimental results}

Fifteen ablations were performed on bovine liver tissue ex vivo using an input power of $50 \mathrm{~W}$ for 3,5 and 10 minutes. Ablated ex-vivo bovine liver tissues are presented in Figure 5. On the pathologic inspection of coagulated regions, different degrees of ablation were noted. The inner zone appears pale, dark and brittle which indicates a high degree of cell death and char; the middle zone appeared pink with coagulated indications and the outer appeared brighter at the boundary between the coagulated part and unaffected part. The size and colour of the ablated zones depend on the type of the antenna, applied input power, and ablation duration. In the figure, (a) charred region is less conspicuous when compared with (b). This may be as a result of a moderate temperature distribution around antenna A. This also resulted in a reduction in backward heating along the antenna shaft because the copper material which made up the outer part of the antenna helped to conduct excessive heat generated during the ablation procedures.

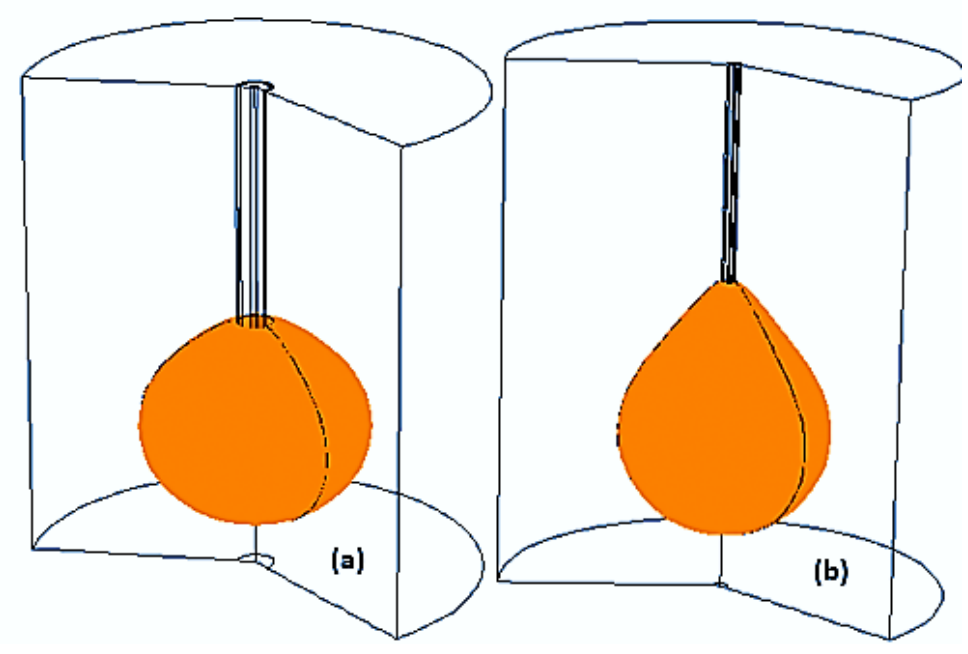

Figure 4. 3-D necrotic regions produce by antenna $A$ and antenna (B) with the corresponding temperature. The black line on each figure denotes a temperature of $52{ }^{\circ} \mathrm{C}$ which is equivalent to the ablated region and where the probability of dead is more than $63 \%$. Sphericity index is higher in (a) than in (b) based on necrotic region produced.

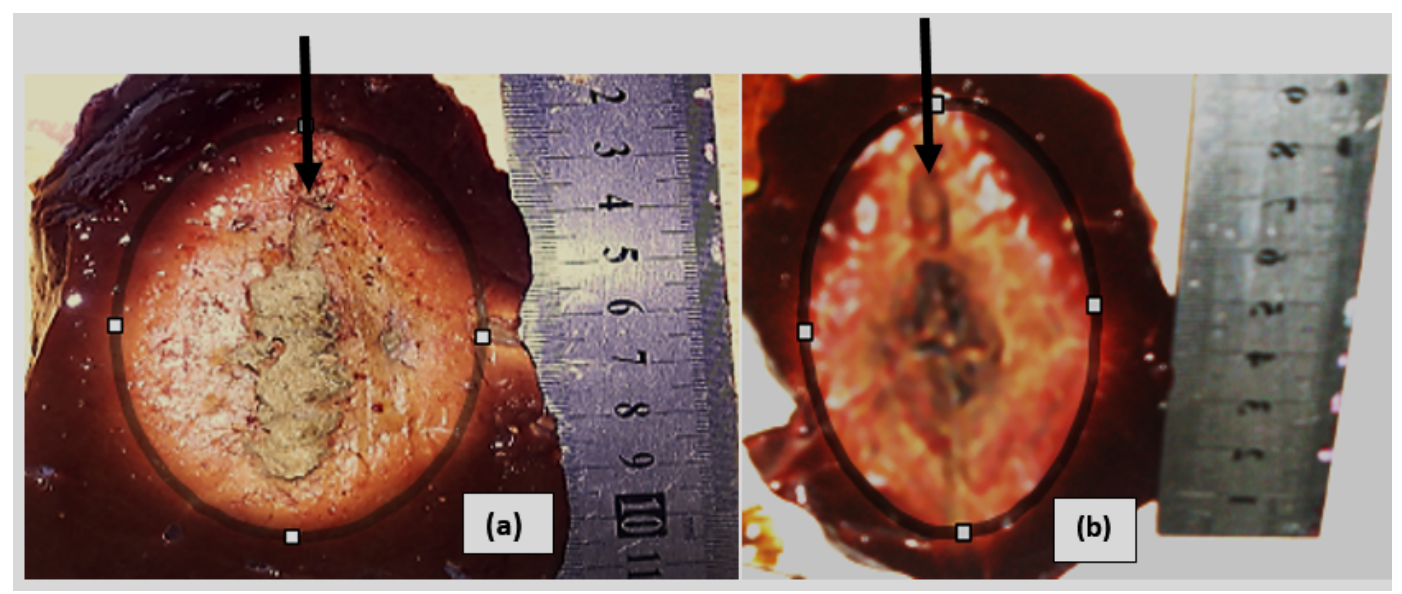

Figure 5. Ex-vivo ablated bovine liver tissues produced (a) by antenna $A$ and (b) by antenna $B$ with an input power of $50 \mathrm{~W}$ for 10 minutes. The black lines in the diagrams define the ablated regions. Lesion produced in (a) was about 4.7 by $4.1 \mathrm{~cm}$ while $4.9 \mathrm{by} 3.8 \mathrm{~cm}$ in (b) with sphericity indices 0.76 and 0.60 respectively. The black arrows on the top of the figures are the point of insertion of the antennas. 
Generally, ablation diameters of coagulated regions increase with the duration of procedures as shown in Figure 6. Antenna A produced a larger ablation diameter than antenna $\mathrm{B}$ at different durations. On average, antenna A produced an ablation diameter of approximately $7.1 \%$ more than that of antenna B. Significant difference was also observed between the ablation diameter of the two antennas $(p=0.04)$. In Figure 7, the ablation length increased as the ablation duration increases. On average, antenna A produced a reduction of $3.9 \%$ in ablation length than Antenna B. This shows that Antenna A has the potential of decreasing backward heating along the antenna shaft by $3.9 \%$. Also, a significant difference exists between the ablation lengths of the two antennas $(p=0.02)$. In Figure 8, antenna A produced more localized microwave energy with high sphericity index than antenna B. On average, antenna A produced $24.2 \%$ in sphericity index more than antenna B. Also, a significant difference exists between the sphericity indices of the two antennas $(\mathrm{p}=0.02)$.

Table 2 shows a summary of simulated and experimental results. From the table, there is no significant difference between the experimental and simulated results for antenna A $(\mathrm{p}=0.94)$ and antenna $\mathrm{B}(\mathrm{p}=0.98)$. The percentage differences between the two parameters are also depicted in the table.

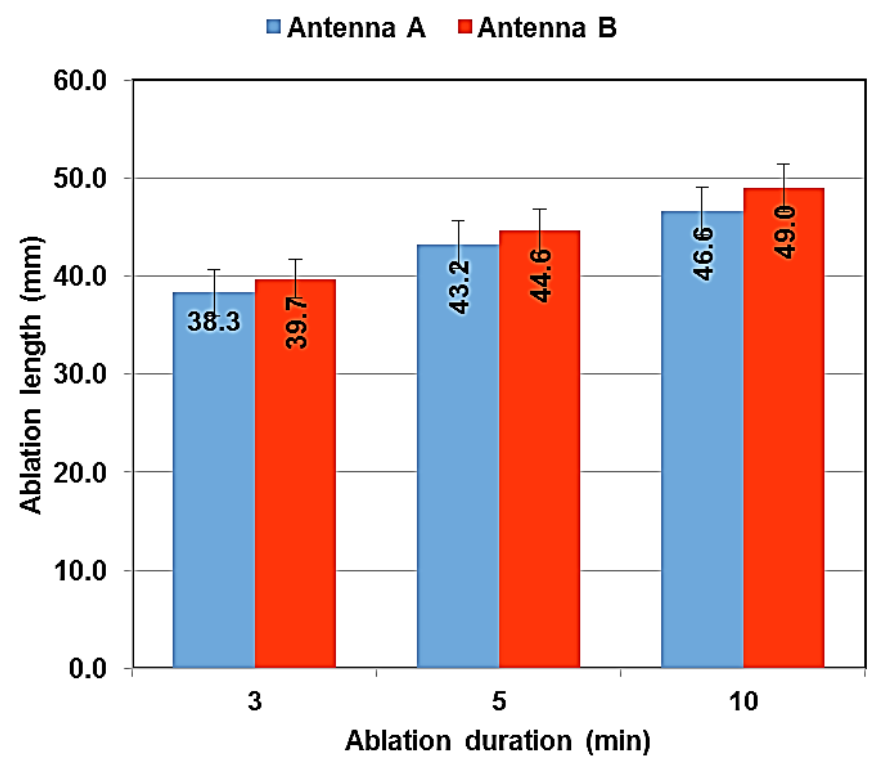

Figure 7. Ablation length versus ablation duration by antennas $\mathrm{A}$ and $B$. The lengths of the ablated regions created along the antenna A longitudinal axis are generally greater than that produced by antenna $B$ which varies with ablation duration.

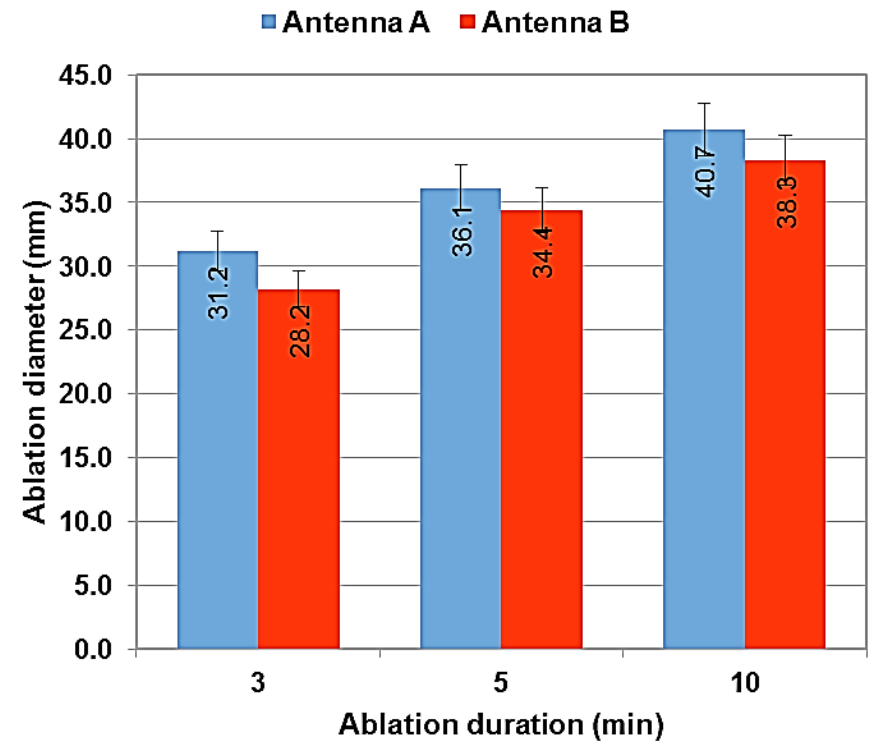

Figure 6. Ablation diameter variation versus ablation duration by antennas $\mathbf{A}$ and $\mathbf{B}$. Increase in ablation duration increases ablation diameter. At each duration Antenna A produces ablated diameter greater than the antenna $B$.

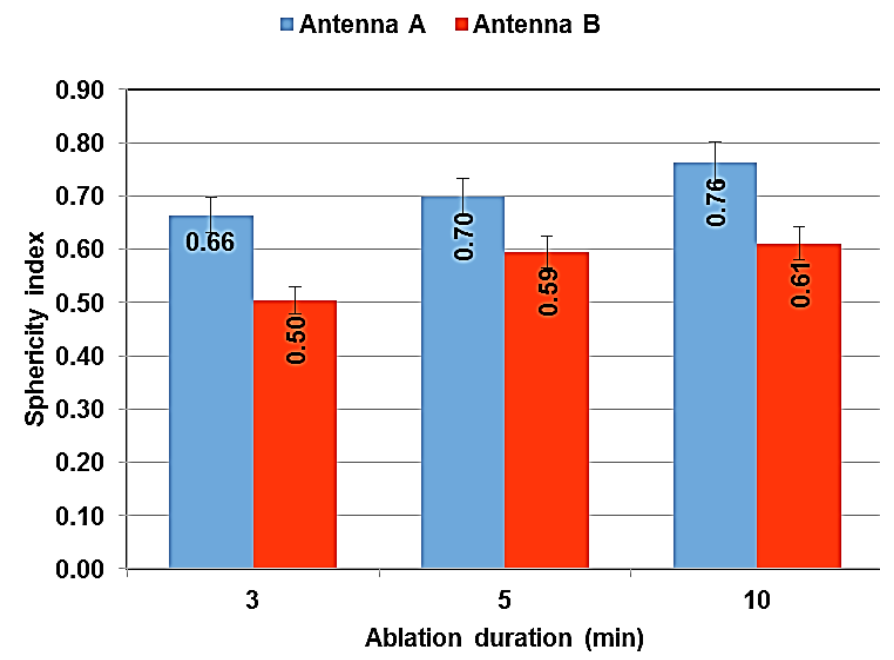

Figure 8. Sphericity index versus ablation duration produced by antennas A and B. Antenna A created a lesion with high sphericity index than antenna $B$. Ablation duration and types of the antennas determined the shape and size of the coagulative regions produced.

Table 2. Comparison between simulated and experimental validation results using the input power of $50 \mathrm{~W}$ for $10 \mathrm{minutes}$

\begin{tabular}{|c|c|c|c|c|c|c|}
\hline \multirow{2}{*}{ Parameter } & \multicolumn{3}{|c|}{ Antenna A } & \multicolumn{3}{|c|}{ Antenna B } \\
\hline & Experiment & Simulation & \%difference & Experiment & Simulation & \%difference \\
\hline ablation Diameter (mm) & 40.7 & 39 & 4.4 & 38.3 & 38.6 & 0.8 \\
\hline ablation length (mm) & 46.7 & 43.6 & 7.1 & 49 & 46.4 & 5.6 \\
\hline Sphericity index & 0.76 & 0.80 & 5.0 & 0.61 & 0.69 & 11.6 \\
\hline p-value & \multicolumn{2}{|c|}{0.94} & \multicolumn{3}{|c|}{0.98} & \\
\hline
\end{tabular}


This paper describes the efficiency of two antennas designed and fabricated from different $50 \Omega$ semi-rigid coaxial cables using numerical simulation and experimental validation methods for microwave ablation tumours. The findings from the study show that antenna A with a thickness of $0.25^{\prime}(6.35$ $\mathrm{mm}$ ) produced better localization of microwave energy in the liver than antenna B of diameter 0.085' (2.21 mm) (Figures 4 \&5). Besides, antenna A created a reduced backward heating and higher ablation diameter and sphericity index than antenna B. Microwave energy is always delivered through an antenna which is a function of its diameter, composite materials, and impedance matching between the tissue and the antenna. The findings in this study also show that there is no significant difference between simulation and experimental validation results (Table 2). The percentage difference in the ablation lengths, ablation diameters and sphericity indices are also minimal. This also is in agreement with the previous studies that computer modeling plays a major role in predicting microwave energy distribution during microwave ablation procedures. ${ }^{2,11-22}$ The applied frequency of the microwave propagating through an antenna and the applied input power is also vital parameters that determine heat distribution pattern in the ablated tissue. Currently, interstitial microwave antennas for percutaneous application are between 1.5 and $2.5 \mathrm{~mm}$ in diameter. Small diameter coaxial cables have been reported to have poor handling power leading to poor performance and heat generation along the antenna shaft. ${ }^{17}$ Antenna prototypes and geometries also play a major role in the localization of microwave energy apart from the complex anatomical structure of an ablated tissue. ${ }^{20}$ Antennas such as monopole, dipole, dual-slot, single-slot, helical and dipole antennas designed and fabricated from a semi-rigid coaxial cable of diameter $0.085^{\prime \prime}$ for MWA are still hindered by some reported shortcomings in the literature. ${ }^{15}$ Antennas, for example, dipole ${ }^{12}$ and dual-slot ${ }^{21}$ have been optimized by the addition of a floating metallic to reduce their backward heating and improve their ability to localize microwave energy into liver tissue. The use of coolant with the antenna to reduce backward heating and improve localization of microwave energy has been proposed. ${ }^{31-32}$ One of the weaknesses of using coolant in reducing backward heating is its potential to absorb microwave energy thereby leading to less energy deposition into the tissue during microwave ablation. The addition of metallic sleeves on these antennas has to an extent reduced backward heating associated with them without a sleeve or choke. ${ }^{12,21}$ The ability of these antennas to withstand high input power with minimal radiative power return loss is still a major concern. ${ }^{17}$ The findings in this study show that antenna fabricated from a thick coaxial cable can withstand high temperature without posing detrimental effects on the adjacent normal tissues. Ablation of tissue with a less charred region (Figure 5) is a piece of evidence that the newly developed antenna can withstand high input power. Also, where ablation diameters exceeding $4.0 \mathrm{~cm}$ is required with high sphericity index and high reduction of backward heating antenna A can be used (Figures 6-8). Besides, antenna A has the potential to be used where sensitive tissues or organs-at-risk preservation are necessary. Research is still ongoing to establish the efficacy of clinical applications of microwave ablation technique in the management of tumours in different organs. ${ }^{30,33}$ A robust antenna to deliver appropriate energy during a microwave ablation procedure is desirable. The choice of an antenna and its application for microwave ablation therapy always depend on clinical indications such as tumor size, tumor location, adjacent organ and tumor morphology. According to this study, the thick antenna will have clinical applications where open surgical approaches are needed despite its larger diameter than the existing ones. More also, antenna developed from thick semi-rigid coaxial cables will be an excellent applicator to ablate large tumour volume with mild temperature with the assurance of preserving adjacent normal organs. In vivo experimentation will be needed to establish the performance of thick antenna to ablate tumour in the liver efficiently since our study was based on simulation and experimental validation methods using ex vivo bovine liver.

\section{Conclusion}

The findings in this study show that microwave power distribution during microwave ablation of biological tissue is affected by antennas' thicknesses. Antennas fabricated from thick semi-rigid coaxial cables have a higher potential of localizing microwave energy during microwave ablation of the liver than the existing ones. Also, the thick antenna provides a larger ablation diameter, higher sphericity index and higher tendency of reducing backward heating along the antenna shaft than the existing antennas. In conclusion, antenna fabricated from thick coaxial cables will have clinical applications where the mild temperature will be required using high input powers for long durations and around organ-at-risks where normal tissue complication probability must be very low.

\section{References}

1. Ahmed M, Brace CL, Lee FT Jr, Goldberg SN. Principles of and advances in percutaneous ablation. Radiology. 2011;258(2):351-69. https://doi.org/10.1148/radiol.10081634

2. Ryan TP, Brace CL. Interstitial microwave treatment for cancer: historical basis and current techniques in antenna design and performance. Int J Hyperthermia. 2017;33(1):3-14. https://doi.org/10.1080/02656736.2016.1214884 
3. Healey TT, March BT, Baird G, Dupuy DE. Microwave Ablation for Lung Neoplasms: A Retrospective Analysis of Long-Term Results. J Vasc Interv Radiol. 2017;28(2):206-211. https://doi.org/10.1016/j.jvir.2016.10.030

4. Maciolek KA, Abel EJ, Best SL, et al. Percutaneous microwave ablation for local control of metastatic renal cell carcinoma. Abdom Radiol (NY). 2018;43(9):2446-2454. https://doi.org/10.1007/s00261-018-1498-Z

5. Izzo F, Granata V, Grassi R, et al. Radiofrequency Ablation and Microwave Ablation in Liver Tumors: An Update. The Oncologist. 2019;24(10):e990-e1005. https://doi.org/10.1634/theoncologist.2018-0337

6. Meloni MF, Chiang J, Laeseke PF, et al. Microwave ablation in primary and secondary liver tumours: technical and clinical approaches. Int J Hyperthermia. 2017;33(1):15-24. https://doi.org/10.1080/02656736.2016.1209694

7. Zhou W, Zha X, Liu X, et al. US-guided percutaneous microwave coagulation of small breast cancers: a clinical study. Radiology. 2012;263(2):364-73. https://doi.org/10.1148/radiol.12111901

8. Ierardi AM, Biondetti P, Coppola A, et al. Percutaneous microwave thermosphere ablation of pancreatic tumours. Gland Surg. 2018;7(2):59-66. https://doi.org/10.21037/gs.2017.11.05

9. Fan QY, Zhou Y, Zhang M, et al. Microwave ablation of malignant extremity bone tumors. Springerplus. 2016;5(1):1373. https://doi.org/10.1186/s40064-016-3005-8

10. Simon CJ, Dupuy DE, Mayo-Smith WW. Microwave ablation: principles and applications. Radiographics. 2005;25:S69-83. https://doi.org/10.1148/rg.25si055501

11. Bertram JM, Yang D, Converse MC, et al. Antenna design for microwave hepatic ablation using an axisymmetric electromagnetic model. Biomed Eng Online. 2006;9:1-9. https://doi.org/10.1186/1475-925X-5-15

12. Yang D, Bertram JM, Converse MC, et al. A floating sleeve antenna yields localized hepatic microwave ablation. IEEE Trans Biomed Eng. 2006;53(3):533-7. https://doi.org/10.1109/TBME.2005.869794

13. Luyen H, Hagness SC, Behdad N. A balun-free helical antenna for minimally invasive microwave ablation. IEEE Trans Antennas Propag. 2015;63:533-65. https://doi.org/10.1109/TAP.2015.2389223

14. Brace CL. Dual-slot antennas for microwave tissue heating: Parametric design analysis and experimental validation. Med Phys. 2011;38(7):4232-4240. https://doi.org/10.1118/1.3601019

15. Bertram JM, Yang D, Converse MC, et al. A review of coaxial-based interstitial antennas for hepatic microwave ablation. Crit Rev Biomed Eng. 2006;34:187-213. https://doi.org/10.1615/critrevbiomedeng.v34.i3.10

16. Ibitoye AZ, Orotoye T, Nwoye EO, Aweda MA. Analysis of efficiency of different antennas for microwave ablation using simulation and experimental methods Egypt J Basic Appl Sci. 2018;5:24-30. https://doi.org/10.1016/j.ejbas.2018.01.005

17. Brace CL. Microwave Tissue Ablation: Biophysics, technology, and applications. Crit Rev Biomed Eng. 2010;38(1):65-78. https://doi.org/10.1615/critrevbiomedeng.v38.i1.60

18. Lubner MG, Brace CL, Hinshaw JL, Lee Jr FT. Microwave tumor ablation: Mechanism of action, clinical results, and devices. J Vasc Interv Radiol. 2010;21:S192-S203. https://doi.org/10.1016/j.jvir.2010.04.007

19. Prakash P. Theoretical modeling for hepatic microwave ablation. Open Biomed Eng J. 2010;4:27-38. https://doi.org/10.2174/1874120701004020027

20. Fallahi H, Prakash P. Antenna Designs for Microwave Tissue Ablation. Crit Rev Biomed Eng. 2018;46(6):495-521. https://doi.org/10.1615/CritRevBiomedEng.2018028554

21. Ibitoye AZ, Nwoye EO, Aweda MA, et al. Optimization of dual-slot antenna using floating metallic sleeve for microwave ablation. Med Eng Phys. 2015;37(4):384-91. https://doi.org/10.1016/j.medengphy.2015.01.015

22. Hand JW. Modelling the interaction of electromagnetic fields (10 MHz-10 GHz) with the human body: methods and applications. Phys Med Biol. 2008;53(16):R243-R286. https://doi.org/10.1088/0031-9155/53/16/R01

23. Deshazer G, Prakash P, Merck D, Haemmerich D. Experimental measurement of microwave ablation heating pattern and comparison to computer simulations. Int J Hyperthermia. 2017;33(1):74-82. https://doi.org/10.1080/02656736.2016.1206630

24. Chiang J, Wang P, Brace CL. Computational modelling of microwave tumour ablations, Int J Hyperthermia. 2013;29(4):308-317. https://doi.org/10.3109/02656736.2013.799295

25. Chiang J, Hynes K, Bedoya M, Brace CL. A dual-slot microwave antenna for more spherical ablation zones: Ex vivo and in vivo validation. Radiology. 2013;268(2):382-389. https://doi.org/10.1148/radiol.13122128

26. COMSOL Multiphysics users' guide. Electromagnetic module and heat transfer module, Version 4.4; www.comsol.com/models

27. Hasgall PA, Di Gennaro F, Baumgartner C, et al. IT'IS Database for thermal and electromagnetic parameters of biological tissues. Version 4.0, May 15, 2018. Accessed 05 June 2019. https://doi.org/10.13099/VIP21000-04-0

28. Andreuccetti D, Fossi R, Petrucci C. An Internet resource for the calculation of the dielectric properties of body tissues in the frequency range $10 \mathrm{~Hz}-100 \mathrm{GHz}$. IFAC-CNR, Florence (Italy), 1997. Based on data published by C. Gabriel et al. in 1996. [Online]. Available: http://niremf.ifac.cnr.it/tissprop 
29. Hines-Peralta AU, Pirani N, Clegg P, et al. Microwave Ablation: Results with a $2.45 \mathrm{GHz}$ Applicator in vitro Bovine and in vivo Porcine Liver. Radiology. 2006;239(1):94-102. https://doi.org/10.1148/radiol.2383050262

30. Ruiter SJS, Heerink WJ, de Jong KP. Liver microwave ablation: a systematic review of various FDA-approved systems. Eur Radiol. 2019;29(8):4026-4035. https://doi.org/10.1007/s00330-018-5842-z

31. Zhou W, Liang M, Pan H, et al. Comparison of ablation zones among different tissues using 2450-MHz cooled-shaft microwave antenna: results in ex vivo porcine models. PloS One. 2013;8(8):e71873. https://doi.org/10.1371/journal.pone.0071873

32. Kuang M, Lu MD, Xie XY, et al. Liver cancer: increased microwave delivery to ablation zone with cooled-shaft antennaexperimental and clinical studies. Radiology. 2007;242(3):914-924. https://doi.org/doi.10.1148/radiol.2423052028

33. Ibitoye AZ, Nwoye EO, Aweda MA, et al. Microwave ablation of ex vivo bovine tissues using a dual-slot antenna with a floating metallic sleeve, Int J Hyperthermia. 2016;32(8): 923-930 https://doi.org/10.1080/02656736.2016.1211323 